\title{
Is percutaneous cement augmentation sufficient for all kinds of vertebral osteoporotic fractures?
}

\author{
Oguz Cebesoy • Unsal Baylar
}

Received: 1 October 2014 / Accepted: 18 October 2014 / Published online: 31 October 2014

(C) SICOT aisbl 2014

I read with great interest the original article by Saracen and Kotwica [1] entitled "Treatment of multiple osteoporotic vertebral compression fractures by percutaneous cement augmentation" published recently in your most valuable journal. In this article, the authors concluded that vertebroplasty should be seriously taken into account as a primary method of treatment in patients with multiple osteoporotic vertebral compression fractures.

I have many questions concerning the procedure:

1. The authors augment only two vertebrae but never two adjacent ones. The first procedures were performed in the most painful areas. A total of 146 patients had three or more fractured vertebrae; thus how do the authors decide which are the painful vertebrae? According to the clinical or radiological examination? Radiologically, the most compressed one may not be painful. It may be healed with fibrosis. I believe the indication for percutaneous vertebroplasty and decision-making for the exact number of levels to be cemented is biased by being subjected to individual surgeon's preference instead of an underlying standardized study protocol for diagnostic screening and surgical indication.

2. It was stated that of the 160 patients during a 24-month follow up, new fractures appeared in three vertebrae. Vertebroplasty does not treat patients with osteoporosis; it is only palliative treatment. Do these patients use any additional antiosteoporotic medications?

3. Were all patients non-responsive to conventional treatments (e.g. pain killers, bed rest, bisphosphonate treatment, brace)?

4. The authors also fail to classify the fracture severity of each affected level, anatomy (1- vs 2- vs 3-column fractures) and grading of associated neurological impairment (e.g. ASIA scale). Why is the same procedure performed for all fractures? Why not choose the kyphoplasty technique for some fractures (i.e. more severe fractures may need a vertebral body height restoration procedure)?

5. Does the patient have any radiculopaty or myelopathy? Disintegration of the posterior canal on MRI? Or any bleeding disorder? Are these patients excluded from the study?

6. What was the radiation time exposure? And what was the refracture rate at the level of 594 augmented vertebrae?

\section{Reference}

1. Saracen A, Kotwica Z (2014) Treatment of multiple osteoporotic vertebral compression fractures by percutaneous cement augmentation. Int Orthop. doi:10.1007/s00264-014-2470-3 